\title{
Weitere Beiträge zur Chemie der Homogentisinsäure.
}

\author{
I. Mitteilung. \\ Von
}

Carl Th. Mörner.

(Der Redaktion zugegangen am 27. März 1912.)

Inhalt.

I. Benzochinonessigsäure, das nächste Oxydationsprodukt der Homogentisinsäure. 1. Darstellung usw. 2. Eigenschaften und Reaktionen. a) Physikalische Beschaffenheit. b) Säurecharakter. c) Chinonsubstanzcharakter. d) Verhalten in wässeriger Lösung. e) Verhalten bei Erhitzung (in Substanz). f) Einige sonstige Reaktionsverhältnisse.

II. Verhalten der Homogentisinsäure in Hinsicht der Ghinhydronbildung. 1. Orientierende Versuche. 2. Darstellung des seigenen. Chinhydrons der Homogentisinsäure. 3. Versuch, egemischte Chinhydrone von Homogentisinsäure usw. darzustellen. III. Verhalten der Homogentisinsäure gegen Ferrichlorid (bei gew. Temperatur).

\section{Benzochinonessigsäure, das nächste Oxydationsprodukt der Homogentisinsäure.}

Bei vorbereitenden Versuchen mit einer Homogentisinsäuresynthese, die, wenn die Reaktion in der beabsichtigten Richtung verlief, in erster Linie Benzochinonessigsäure ergeben sollte, empfand ich das Bedürfnis einer näheren Kenntnis der Eigenschaften dieser Verbindung, um, darauf gestützt, leichter mit der weiteren Bearbeitung der Reaktionsmischung zurechtzukommen. Zwar bin ich schon früher ${ }^{1}$ ) mit gewissen Derivaten der Benzochinonessigsäure, ihrem Dianilid, Di-p-toluidid usw., in Berührung gekommen, bezüglich der Säure selbst ermangelte ich aber jeder eigenen Erfahrung. Auch bei einer eingehenden

1) Diese Zeitschrift, Bd. 69 (1910), S. 329. 
Literaturprüfung war kein einziger Aufschluß hierüber zu erhalten. Allem nach zu urteilen ist diese, doch verhältnismäßig einfach konstituierte, aus der Natur nicht bekannte Verbindung bisher nicht auf synthetischem Wege dargestellt bezw. beschrieben worden. Unter solchen Umständen entschloß ich mich, zu versuchen, die genannte Verbindung darzustellen, und zwar, von der Homogentisinsäure ausgehend, durch Chromatoxydation derselben in saurer Flüssigkeit.

\section{Darstellung usw.}

Von Homogentisinsäure ${ }^{1}$ ) wurden Portionen von 10 bezw. $20 \mathrm{~g}$ in Arbeit genommen, unter Beobachtung folgender Relation in der Reaktionsmischung, pro $1 \mathrm{~g}$ Homogentisinsäure gerechnet:

$\begin{array}{ll}\text { Wasser } & 20 \mathrm{ccm} \\ \text { konzentrierte Schwefelsäure } & 2 \mathrm{~g} \\ \left.\text { gesättigte Natriumbichromatlösung }{ }^{2}\right) & 2 \mathrm{ccm}\end{array}$

Die Homogentisinsäure wird in dem zuvor mit der Schwefelsäure versetzten Wasser gelöst. Nach Abkühlung der Flüssigkeit auf ca. $\pm 0^{0}$ C. wird die Bichromatlösung aus einer Bürette tropfenweise und unter Umrühren, jedoch nicht mehr als $2 \mathrm{ccm}$ nacheinander hinzugesetzt; $z$ wischen jedem solchen Zusatz wird die Mischung durch Einpacken des Gefäßes in Schnee abgekühlt. Hierbei beobachtet man das Auftreten goldglänzender, dünner

1) Die für die in diesem Aufsatz beschriebenen Versuche erforderlichen beträchtlichen Homogentisinsäuremengen wurden aus (nach Garrod aus Alkaptonharn dargestelltem) Bleisalz durch Umsetzen desselben (bei Zimmerwärme, unter fleißigem Umschütteln) mittels berechneter Menge doppelter Normalschwefelsäure (175 ccm pro $100 \mathrm{~g}$ Bleisalz), Abfiltrierung von Bleisulfat, Konzentrierung des schon an sich hochprozentigen Filtrats im Vakuum (bei Zimmerwärme) bis zu reichlicher Auskrystallisierung, kräftiges Auspressen der Krystalle und Auflösen in wenig Wasser (Filtrieren); danach erneutes Konzentrieren im Vakuum, bis nur eine ganz geringe Menge Mutterlauge übrig ist, Auspressen, Trocknen bei Zimmerwärme über Schwefelsäure. Die Säure ist, auf diese Weise dargestellt, fast farblos und frei von Lactonbeimischung, was der Regel nach nicht der Fall bei Präparaten ist, die nach den in den Handbüchern üblichen Vorschriften bereitet sind, welch letztere sich auf die Zersetzung des Bleisalzes mittels Schwefelwasserstoff usw. gründen.

2) Spez. Gewicht ca. 1,75. 
Krystalltafeln in der dunkelgefärbten Flüssigkeit. Nach eintägigem Stillstehn bei ca. $\pm 0^{0}$ wird die Reaktionsmischung filtriert, und die Krystallmasse mit schneegekühltem Wasser (jedesmal kleine Portionen) gewaschen, bis das Waschwasser nicht mehr Schwefelsäurereaktion mit $\mathrm{BaCl}_{2}$ gibt; Auspressen und Trocknen im $\mathrm{H}_{2} \mathrm{SO}_{4}$-Exsikkator. Ausbeute in verschiedenen Versuchen 41-45\% der angewandten Menge Homogentisinsäure (Fraktion A). Durch Schütteln der Mutterlauge + Waschwasser mit Äther ( 4 mal, jedesmal $1 / 2$ Volumen Äther) und Verdunstung des mittels $\mathrm{Na}_{2} \mathrm{SO}_{4}$ vom Wasser befreiten Äthers bei Zimmerwärme wird eine weitere beträchtliche Menge der schön krystallisierten Substanz, 23-26\% $\%^{1}$ ) der Homogentisinsäuremenge entsprechend, erhalten (Fraktion B).

Das auf den beiden Wegen erhaltene Rohprodukt wurde zunächst jedes für sich umkrystallisiert, wonach durch wiederholte Umkrystallisierung noch eine weitere Anzahl Präparate hergestellt wurden. In Anbetracht der bei der Darstellung zutage getretenen relativ geringeren Löslichkeit der Substanz in kaltem Wasser möchte man geneigt sein, in warmem Wasser ein gutes Medium zur Umkrystallisierung zu erblicken; es ist indessen vollkommen unanwendbar, da die Substanz bei Behandlung damit zerfällt (siehe unten S. 314). Dagegen läßt sich die Substanz mit Leichtigkeit und mit guter Ausbeute aus gewissen organischen Lösungsmitteln umkrystallisieren. Folgende drei Verfahren sind hierzu angewandt worden:

a) Die Substanz wird in Aceton $\left(10 \mathrm{ccm}^{2}\right)$ ) gelöst, Chloroform $(10 \mathrm{ccm})$ hinzugesetzt, eine eventuelle Trübung abfiltriert, Ligroin $(40 \mathrm{ccm}$ ) hinzugesetzt (unmittelbar beginnende Ausscheidung von Krystallen); nach einem Tage (bei Zimmerwärme) wird der Krystallniederschlag auf das Filter genommen, ausgepreßt und erst in der Luft an kühler Stelle einige Tage lang, dann im $\mathrm{H}_{2} \mathrm{SO}_{4}$-Exsikkator bis zu konstantem Gewicht, das sich binnen einiger Tage einstellt, getrocknet.

1) Ungefähr die Hälfte davon wird aus dem 1. Ätherextrakt gewonnen; aus dem 4. nur eine ganz unbedeutende Menge. Gesamtausbeute also: ca. $65-70 \%$.

2) Die Zahlen beziehen sich auf 1 g Substanz. 
b) Aus dem Filtrat von a) werden, durch Anwendung gelinder Wasserbadwärme, Aceton und Chloroform, sowie ein Teil des Ligroins entfernt, wobei wieder eine ziemlich reichliche Ausscheidung von Krystallen stattfindet, die sofort auf das Filter genommen werden usw.

c) Bei Wasserbadwärme wird eine gesättigte Benzollösung der Substanz bereitet, die noch warm filtriert wird (Krystalle scheiden sich wäbrend des Abkühlens aus). Weitere Behandlung der Krystalle wie oben.

Die dargestellten Präparate sind nachstehend nach dem bei der Umkrystallisierung angewandten Verfahren gruppiert:

\begin{tabular}{|c|c|c|c|c|c|c|}
\hline & & & $\begin{array}{l}\text { Mol.- } \\
\left.\text { Gew. }{ }^{1}\right)\end{array}$ & $\begin{array}{c}\text { C- } \\
\left.\text { Gehalt }{ }^{2}\right) \\
\%\end{array}$ & $\begin{array}{c}\mathrm{H}- \\
\left.\text { Gehalt }{ }^{2}\right) \\
\%\end{array}$ & $\begin{array}{l}\text { Schmelz- } \\
\text { punkt }^{3} \text { ) } \\
{ }^{\circ} \mathrm{C} .\end{array}$ \\
\hline \multirow[t]{4}{*}{ a) } & Präparat & I... & 169 & $\left.58,05^{4}\right)$ & 3,77 & 122 \\
\hline & > & II . . . . . & 168 & 57,94 & 3,92 & 124 \\
\hline & , & III . . . . & 167 & - & - & 126 \\
\hline & > & IV . . . & 166 & - & - & 126 \\
\hline \multirow[t]{3}{*}{ b) } & , & v . . . & 166 & 57,52 & 3,68 & 131 \\
\hline & & ... & 167 & - & - & 130 \\
\hline & > & VII . . . . & 167 & 58,09 & 3,76 & 129 \\
\hline \multirow[t]{2}{*}{ c) } & , & VIII . . . & 167 & - & - & 131 \\
\hline & $>$ & IX . . . . & - & - & - & 130 \\
\hline \multicolumn{3}{|c|}{ Berechnet für $\mathrm{C}_{8} \mathrm{H}_{6} \mathrm{O}_{4}$} & 166 & 57,81 & 3,65 & - \\
\hline
\end{tabular}
phthalein).

1) Bestimmt durch Titrierung mit n/10-Alkali (Indikator: Phenol-

2) Die in diesem Aufsatz angeführten C- und H-Bestimmungen sind von Herrn Dr. H. W eil, München, ausgeführt worden.

s) Bei Bestimmung des Schmelzpunktes, richtiger des Zersetzungspunktes (reichliche Gasentwickelung), wurde das Kapillarrohr in das zuvor auf $115-120^{\circ}$ erhitzte Schwefelsäurebad hinabgetaucht.

4) Präparat I. $0,1597 \mathrm{~g}$ ergaben $0,3399 \mathrm{~g} \mathrm{CO}_{2}, 0,0537 \mathrm{~g} \mathrm{H}_{2} \mathrm{O}$.

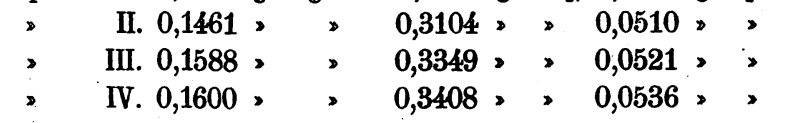


Stellt man die gefundenen Molekulargewichte und die C- und H-Werte mit dem vorhandenen ausgesprochenen Charakter als Säure und als Chinonsubstanz (siehe unten) zusammen, so kann kein Zweifel herrschen an der Identität der dargestellten Substanz mit der berechneten, d. h. mit Benzochinonessigsäure, $\mathrm{C}_{6} \mathrm{H}_{3} \mathrm{O}_{2} \cdot \mathrm{CH}_{2} \cdot \mathrm{COOH}$ oder:

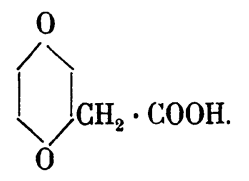

Der innerhalb der Gruppe a) der Präparate beobachtete niedrigere Schmelzpunkt dürfte darauf beruhen, daß diese - im Gegensatz zu den übrigen - ohne Erhitzung bereiteten Präparate auf irgend eine Weise eine geringe Menge Aceton aufnehmen und auch nach länger dauernder Exsikkatorbehandlung trotz eingetretener Gewichtskonstanz hartnäckig dieselbe festhalten (bei Destillierung mit Wasser zeigt eine zuerst übergehende, kleinere Fraktion deutliche Reaktion bei Liebens Jodoformprobe). Hierzu kommt, daß innerhalb derselben Gruppe die beiden Präparate, Nr. I und II, die die niedrigsten Schmelzpunkte aufweisen, das erste Umkrystallisierungsprodukt von Fraktion A bezw. Fraktion B darstellen, d. h. - im Gegensatz zu den beiden anderen - nur einmal umkrystallisiert sind.

\section{Eigenschaften und Reaktionen.}

Ganz allgemein läßt sich von der Benzochinonessigsäure sagen, daß mit der Substanz - vom Laborationsgesichtspunkt aus - ebenso angenehm zu arbeiten ist, wenn man indifferente, organische Lösungsmittel anwendet, unter Vermeidung von unnötig starker Erhitzung, wie sie (infolge von Destruktion) unhantierlich in wasserhaltigem Medium ist, zumal wenn sich höhere Temperatur dazu gesellt. Dieser Umstand dürfte wesentlich schuld daran sein, daß diese - wie bereits erwähnt verhältnismäßig so einfach konstruierte Verbindung bisher nicht in den Gesichtskreis des chemischen Forschers getreten ist. 
a) Physikalische Beschaffenheit.

Die Substanz krystallisiert in Form von dünnen, rhomboedrischen oder quadratischen Tafeln, die der Farbe und dem Glanze nach an Bleijodid oder «Mussivgold» erinnern. Im Gegensatz zum Benzochinon nicht flüchtig, ${ }^{1}$ ) ermangelt es jeder Andeutung von Geruch. Der Geschmack ist schwach sauer, danach bitter. Lösungen der Substanz besitzen, je nach der Konzentration, gelbe-rotgelbe, gelbrote-braunrote Farbe. In Wasser ist die Substanz ziemlich löslich, wenn auch nicht in gewöhnlichem Sinne leichtlöslich (vgl. S. 308 über die Darstellung); in solcher Lösung tritt sehr bald Destruktion ein (siehe unten S. 314). Was die allgemeiner gebräuchlichen, organischen Lösungsmittel betrifft, so läßt sich die Substanz als leichtlöslich bezeichnen in Äthylalkohol, Äthyläther, Äthylacetat, Methylalkohol, Aceton, Epichlorhydrin, Anilin und Eisessig; ${ }^{2}$ ) ziemlich löslich ist sie in Amylalkohol; bei Zimmerwärme fast oder ganz unlöslich in Chloroform, Tetrachlormethan, Benzol, Toluol, Xylol, Petroleumäther, Ligroin und Kohlendisulfid.

Der Schmelz-(Zersetzungs-) Punkt für reine Benzochinonessigsäure liegt bei ca. $+130^{\circ}$ C. (s. oben S. 309) - der für

1) Siehe S. 320 .

8) Auch in Pyridin löst sich die Substanz mit Leichtigkeit, binnen weniger als einer Minute aber erstarrt die braunrote Lösung zu einem Magma, gebildet aus farblosen, glänzenden Krystallen (Rosetten von kurzen Prismen), die sich bei Zusatz, selbst in reichlicher Menge, weder von Pyridin noch von absolutem Alkohol lösen. Orientierende Versuche mit der isolierten Substanz zeigen, daß dieselbe nicht, wie man zunächst wohl annehmen könnte, aus dem Pyridinsalz der Benzochinonessigsäure besteht, sondern aus einem komplizierteren Reaktionsprodukt - $\mathbf{u}$. a. süßschmeckend, ziemlich leichtlöslich in Wasser mit approximativ neutraler Reaktion; mit $\mathrm{FeCl}_{3}$ indigoblaue, bald vorübergehende Färbung ergebend; ammoniakalische Silberlösung unmittelbar reduzierend, bei Kochen mit Natronlauge intensiven Pyridingeruch von sich gebend; der allgemeinen Chinonreaktionen ermangelnd - eine Substanz, die offenbar ein näheres Studium verdient. Die der Benzochinonessigsäure nahestehenden Substanzen Benzochinon, Benzohydrochinon, Homogentisinsäure und ihre Lactone verhalten sich in der fraglichen Hinsicht gegenüber Pyridin indifferent. 
Benzochinon bekanntlich bei $+116^{\circ}$, der für Homogentisinsäure bei $+147^{\circ}$.

\section{b) Säurecharakter.}

Dieser tritt sehr deutlich nicht nur durch den Geschmack hervor, sondern auch in dem Verhalten zu acidi-alkalimetrischen Indikatoren; so wird z. B. Kongopapier blaugefärbt. In Übereinstimmung hiermit kann die Substanz acidimetrisch titriert werden (z. B. mit Phenolphthalein als Indikator), welches Verhältnis den auf S. 309 angegebenen Molekulargewichtsbestimmungen zugrunde liegt.

\section{c) Chinonsubstanzcharakter.}

Schon 1867 fand Schaer, ${ }^{1}$ ) daß gewöhnliches Chinon, d. h. Benzochinon, «ozonidischen» Charakter besitzt, der sich in seinem Vermögen äußert, Guajaktinktur und angesäuerte Jodkaliumstärkekleister blau zu färben. Auf das Vermögen des Benzochinons (und anderer untersuchten Chinonsubstanzen), in saurer Lösung Jod aus Kaliumjodid nach dem Schema:

$$
\mathrm{C}_{6} \mathrm{H}_{4} \mathrm{O}_{2}+2 \mathrm{HCl}+2 \mathrm{KJ}=\mathrm{C}_{6} \mathrm{H}_{6} \mathrm{O}_{2}+2 \mathrm{KCl}+2 \mathrm{~J}
$$

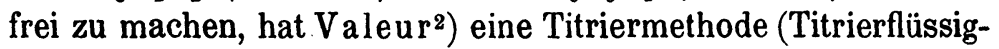
keit: n/10-Natriumhyposulfit) zur quantitativen Bestimmung von Chinonsubstanzen, bezw. zur Bestimmung ihres Molekulargewichts gegründet. Auch bei der von Liebermann ${ }^{3}$ ) angegebenen qualitativen Chinonreaktion kommt derselbe *ozonidische" Charakter zum Ausdruck. Die fragliche Reaktion wird von Liebermann folgendermaßen beschrieben: «Setzt man zu 1-2 Tropfen einer farblosen, alkoholischen Hydrocörulignonlösung wässerige Chinonlösung, so färbt sich die Mischung sofort gelbrot und gleichzeitig scheiden sich unter Wiederentfärbung der Lösung die stahlblauen, schillernden Nadeln von Cörulignon aus». Liebermann bemerkt ferner: "Nicht alle Chinone reduzieren sich gleich leicht, und daher gibt $\mathbf{z}$. B. Thymochinon die Reaktion nicht oder ungenügend.»

1) Zeitschr. f. Biologie, Bd. 37 (1899), S. 320.

2) Compt. rend., Bd. 129 (1899), S. 552.

$\left.{ }^{3}\right)$ Berichte der Deutsch. chem. Ges., Bd. 10 (1877), S. 1615. 
Was nun Benzochinonessigsäure betrifft, so hat sie genau dasselbe Verhalten wie Benzochinon selbst gezeigt, d. h. sie reagiert kräftig positiv bei allen 3 angegebenen Reaktionen.

Bei Versuchen, das Molekulargewicht nach Valeur zu bestimmen, sind Werte (167-168) erhalten worden, die mit den auf acidimetrischem Wege festgestellten nabe übereinstimmen.

Das für Liebermanns Reaktion erforderliche Hydrocörulignon wurde, da es nicht im Handel erhältlich war, von mir aus rohem Holzessig (Acetum pyrolignosum) nach dem von L. ${ }^{1}$ ) angegebenen Verfahren dargestellt. Bei Arbeit in kleinem Maßstabe, wie es hier der Fall war (Ausgangsmaterial: $500 \mathrm{~g}$ roher Holzessig), läuft man indessen bei strenger Befolgung von L.'s Vorschrift Gefahr, das Hydrocörulignon zu averlieren`, nämlich infolge seiner nicht vollständigen Unlöslichkeit in der HCl-sauren mit $\mathrm{Zn}$ behandelten Flüssigkeit. In dem nach vollendeter Reduktion abfiltrierten unbedeutenden Rückstande befand sich die gesuchte Substanz nicht, wohl aber in dem Filtrat, aus dem sie mit Leichtigkeit durch Ausschütteln mit Äther, Verdunstung des Äthers und Umkrystallisierung des Rückstandes aus etwas Alkohol (farblose Krystallnadeln) zu erhalten war. ${ }^{2}$ )

Ein weiteres einfaches Reagens zum Nachweis der kräftig oxydierenden Wirkung der Chinonsubstanzen habe ich in einèr nahezu farblosen, mit Schwefelsäure angesäuerten Lösung von Ferrosulfat + Ammoniumrhodanid gefunden, die unmittelbar intensiv rote Farbe (vom Ferrirhodanid) bei Zusatz von Benzochinon oder Benzochinonessigsäure annimmt.

Eine andere Seite des fraglichen Chinoncharakters der Substanz tritt durch ihr Vermögen zutage, mit ihrer entsprechenden Hydrochinon-(p-Dioxybenzol-)Substanz, d. h. mit Homogentisinsäure, ein typisches Chinhydron zu bilden (genaueres darüber siehe S. $321 \mathrm{ff}$.).

\section{d) Verhalten in wässeriger Lösung.}

In wässeriger Lösung zeigt Benzochinonessigsäure bemerkenswert geringe Resistenz. Die in frisch zubereitetem $\mathrm{Zu}$ -

') Ann. d. Chemie u. Pharmacie, Bd. 169 (1873), S. 221.

2) Beobachtung: In konzentrierter Schwefelsäure löst sich Hydrocörulignon mit orangeroter Farbe; wird ganz wenig Kaliumdichromat zugesetzt, so geht die Farbe in intensives Indigoblau über (Cörulignon gibt, wie L. angibt, mit konzentrierter Schwefelsäure direkt die letztere Farbenreaktion). 
stande klare, rotgelbe Lösung nimmt bei Aufbewahrung immer dunklere, braune bis schwarzbraune Farbe an und scheidet ein fast schwarzes Sediment ab (dies schon bei Zimmerwärme und auch geschützt gegen Tageslicht). Bei Erwärmung tritt die Veränderung weit rascher auf. Aus der so veränderten Lösung werden, bei Ausschütteln mit Äther und Verdunsten des Äthers, schließlich nicht weiter Krystalle von Benzochinonessigsäure erhalten, wohl aber ein Rest, in dem Homogentisinsäure durch Überführung in ihr charakteristisches Bleisalz nachgewiesen werden kann. Schritt für Schritt kann auch die Destruktion der Benzochinonessigsäure bezw. das Auftreten von Homogentisinsäure verfolgt werden durch Prüfung mit Ferrichlorid, indem die Substanz selbst (in frisch zubereiteter wässeriger Lösung) nicht damit reagiert, während Homogentisinsäure bekanntlich eine, wenn auch vorübergehende Blaufärbung ergibt.

Zur weiteren Beleuchtung der diesbezüglichen Verhältnisse seien einige Versuche wiedergegeben.

0,1632 g Benzochinonessigsäure wurden in einer Platinschale mit Wasser übergossen; Erwärmung in Wasserbad während 5 Stunden, wobei verdunstetes Wasser von Zeit zu Zeit wieder ersetzt wurde; schließlich wurde zur Trockenheit eingedunstet und danach eine weitere Stunde erhitzt. Trocknen im $\mathrm{H}_{2} \mathrm{SO}_{4}$-Exsikkator bis zu Gewichtskonstanz. Der glänzende, schwarzbraune, firnisartige Rückstand wog 0,1132 g (entsprechend einem Gewichtsverlust von ca. 31\%). Der Rückstand wurde mit etwas Wasser aus der Schale entnommen, der braunschwarze Bodensatz wurde abfiltriert, das Filtrat mit neutraler Bleiacetatlösung versetzt, aufgekocht, durch Filtrieren von braunen Flocken befreit. Aus dem Filtrat schieden sich bis zum folgenden Tage Krystallnadeln von homogentisinsaurem Blei aus. Das Auftreten eines dunklen Bodensatzes, wie auch der bedeutende Gewichtsverlust zeigen, daß die Destruktion der Substanz nicht nur eine "Selbstreduktion» zu Homogentisinsäure darstellt, sondern daß die Reaktion von weit komplizierterer Natur ist. Ein "Chinongeruch», d. h. ein solcher, wie er dem Benzochinon zukommt, wurde in keinem Stadium dieses Versuches bemerkt. 
Anläßlich des beobachteten Gewichtsverlustes wurde folgender Versuch angestellt.

$0,150 \mathrm{~g}$ Substanz $+30 \mathrm{ccm}$ Wasser wurden aus Kolben mit Kühler destilliert, bis das Volumen des Destillats $15 \mathrm{ccm}$ betrug (der rückständige Kolbeninhalt 1 ) war dunkelbraun und trübe). Das Destillat war farb-, geruch- und geschmacklos, mit nur schwacher Andeutung zu saurer Reaktion; es ergab nicht Blaufärbung mit angesäuertem Jodkaliumstärkekleister und reduzierte nicht ammoniakalische Silberlösung. Es ließ sich also auch bei dieser Versuchsanordnung kein Benzochinon nachweisen, gleichwie auch kein anderes charakterisierbares Zersetzungsprodukt in dem Destillat angetroffen wurde.

Man könnte vielleicht sich vorstellen, daß die der freien Säure zukommende Nejgung zu Zerfall in wässeriger Lösung sich nicht geltend machen würde, wenn die Säure an Alkali zu neutralem Salz gebunden wäre. Das ist aber nicht der Fall. Eine mit verdünnter Kalilauge sorgfältig neutralisierte, frisch zubereitete wässerige Lösung wurde in 2 gleichgroße Portionen geteilt.

a) Die eine wurde sofort in verdünnte Schwefelsäure gegossen und die Mischung mit Äther ausgeschüttelt, der beim freiwilligen Verdunsten Krystalle vom gewöhnlichen Aussehen der Benzochinonessigsäure gab. Nach Umkrystallisierung wurde der Schmelzpunkt zu $+128^{\circ}$, das Molekulargewicht zu $169^{\circ}$ bestimmt.

b) Die zweite Portion wurde im Vakuum verdunsten gelassen, wobei sie dunkler und dunkler wurde. Aus dem dunkelbraunen, firnisartigen Rückstande konnten, nach Ansäuerung mit verdünnter Schwefelsäure und Ausschütteln mit Äther, keine Krystalle von Benzochinonessigsäure erhalten werden.

e) Verhalten bei Erhitzung (in Substanz).

Auch bei Abwesenheit von Wasser wird die Substanz bei gewöhnlicher Trockenschrankwärme $\left(+105^{\circ}\right)$, ja, bei noch

1) Nach Abkühlung und Filtrieren wurde mit angesäuertem Jodkaliumstärkekleister geprüft: keine Blaufärbung; mit Ferrichloridlösung gab die Flüssigkeit dagegen, in Übereinstimmung mit einer Homogentisinsäurelösung, vorübergehende Blaufärbung. 
niedrigerer Temperatur destruiert. Eine $0,1712 \mathrm{~g}$ wiegende Portion wurde in einen Trockenschrank von erwähnter Temperatur hineingestellt. Binnen einigen Minuten hatte die ursprüngliche, schön gelbe Farbe einer schmutzigen, dunkelgrauen Platz gemacht; nach 20 Minuten erblickte man eine stark aufgeblähte und zusammenhängende, glänzende, pechschwarze Masse. Bei fortgesetzter Trockenschrankbehandlung trat nach 11/2 Stunden Gewichtskonstanz ein; das Gewicht nun: 0,1480 g (entsprechend einem Gewichtsverlust von ca. 14\% $/$ ). Beim Schütteln der Masse mit Wasser erhielt man ungelöst ein kohlenschwarzes Pulver, das nach Trocknen bei $+105^{\circ} 0,0456 \mathrm{~g}$ wog. Dieses löste sich leicht und vollständig in Ammoniak; bei Ansäuerung der Lösung mit Schwefelsäure fielen schwarze Flocken aus.

Auch bei der Erhitzung in Substanz verspürte man in keinem Stadium «Chinongeruch », ebensowenig eine andere deutliche Geruchsqualität.

f) Einige sonstige Reaktionsverhältnisse.

1. Durch Behandlung mit Schwefeldioxyd kann Benzochinonessigsäure in Homogentisinsäure übergeführt werden, ohne daß dabei dunkelgefärbte Nebenprodukte in nennenswerter Menge entstehen.

2. Durch konzentrierte Schwefelsäure werden die Krystalle sofort schwarz gefärbt, wonach sie sich zu einer schmutzig olivfarbenen Flüssigkeit lösen. Konzentrierte Chlorwasserstoffsäure gibt weder bei Zimmerwärme noch bei Kochen Anlaß zu einer Farbenreaktion.

3. Die wässerige Lösung scheidet keinen Niederschlag aus, wenn man Bromdämpfe successiv absorbieren läßt, oder wenn Bromwasser hinzugesetzt wird.

4. Millons Reagens fällt nicht unmittelbar die wässerige Lösung; bei Kochen keine Rotfärbung (bei Abkühlen setzt sich ein hell orangegelber Niederschlag $a b)$.

5. Mit Ferrichloridlösung gibt die wässerige Lösung (frisch zubereitet!) keine Färbung oder Fällung (vgl. S. 314).

6. Neutrale Bleiacetatlösung bewirkt nicht unmittelbar 
einen Niederschlag; später tritt die Destruktion der Substanz ein, sich in reichlicher Entstehung eines schwarzbraunen Bodensatzes äußernd.

7. Silbernitratlösung wird nicht innerhalb einer Stunde bei Zimmerwärme reduziert; bei Kochen entsteht dagegen bald ein Metallspiegel. Ammoniakalische Silberlösung wird momen$\tan$ reduziert.

Bei Zusatz von Fehlingscher Flüssigkeit wird Oxydul ausgeschieden, bei Zimmerwärme binnen $1 / 2$ Stunde, bei Kochen sofort.

Diese Reaktionen, mit den aus S. 312 angegebenen zusammengestellt, zeigen, daß Benzochinonessigsäure - in Übereinstimmung z. B. mit Benzochinon und Wasserstoffsuperoxyd - in ausgesprochenem Grade sowohl Reduktions- als 0xydationsvermögen besitzt.

8. Eine schöne Farbenreaktion, die der Benzochinonessigsäure mit dem Benzochinon gemeinsam ist, und die meines $\mathrm{W}$ issens bisher nicht angegeben worden ist, erhält man, wenn man die wässerige Lösung zuerst mit Jodjodkaliumlösung, danach ganz vorsichtig mit Natronlange ${ }^{1}$ ) versetzt: kirschrote Färbung, bei weiterem Natronlaugenzusatz übergehend in olivgrüne.

9. Ein von Raciborski²) für Benzochinon angegebenes Reaktionsverhalten - Entstehung intensiver Rotfärbung, wenn Benzochinon in wässeriger Lösung auf Aminosäuren oder gewisse Proteinstoffe einwirkt - habe ich zunächst bezüglich einiger Aminosäuren nachgeprüft, wobei ich das angegebene Verhältnis habe konstatieren können. Die Mischungen ließ ich bei Zimmerwärme 24 Stunden lang stehen:

$\left.\begin{array}{l}\text { Glykokoll } \\ \text { Alanin (r-) } \\ \text { Leucin (r-) }\end{array}\right\}$ kirschrot,

Tyrosin ( $r-$ ) rot (ungefähr dieselbe Qualität wie bei

»(l-) $\}$ Millons Reaktion),

1) Die Reaktion gelingt nicht mit Natriumcarbonat.

2) Anz. Akad. Wiss. Krakau, 1096, S. 553 (nach Referat im Chem. Zentralbl., 1907, Bd. 1, S. 1595). 
$\left.\begin{array}{rr}\text { Dibromtyrosin }(\mathbf{r}-) \\ (\mathbf{l})\end{array}\right\}$ rotviolett.

Benzochinonessigsäure, an denselben Objekten geprüft, gibt indessen keine solche Reaktion (nur Braunfärbung und Ausscheidung eines graubraunen Bodensatzes).

II. Verhalten der Homogentisinsäure in Hinsicht der Chinhydronbildung.

Unter Chinhydronen versteht man bekanntlich lockere Doppelverbindungen, dadurch entstanden, daß 1 Molekül Hydrochinon-(p-Dioxybenzol-)Substanz sich zu 1 Molekül Chinonsubstanz addiert. So wird z. B das «gewöhnliche» Chinhydron durch die Addition des gewöhnlichen Hydrochinons (= Benzohydrochinons) zu der demselben entsprechenden Chinonsubstanz, d. h. Benzochinon, gebildet, weshalb das gewöhnliche Chinhydron als das «eigene» Chinhydron des Benzohydrochinons bezw. Benzochinons bezeichnet werden kann. In einem Chinhydron, entstanden durch Addition z. B. von Benzohydrochinon zu einer demselben *fremden ${ }^{2}$ Chinonsubstanz, z. B. $\alpha$-Naphthochinon, haben wir ein Beispiel für sog. "gemischte» Chinhydrone (Urban). ${ }^{1}$ ) Die Chinhydrone sind bekannt als dunkelgefärbte, gut krystallisierende, metallisch glänzende Substanzen; sie sind leicht dissoziierbar besonders in Wasser, weshalb bei ihrer Darstellung nur ausnahmsweise (z. B. betreffs der gewöhnlichen Chinhydrone) Wasser als Lösungsmittel für die beiden Konstituenten angewandt werden kann.

Eine Angabe über Chinhydronbildung aus Homogentisinsäure (= Benzohydrochinonessigsäure) habe ich in der Literatur nicht finden können. Als durch die nun geschehene Darstellung der Benzochinonessigsäure eine Möglichkeit vorlag, zu versuchen, das «eigene» Chinhydron der Homogentisinsäure darzustellen, wurde die Gelegenheit hierzu benutzt. Im Zusammenhang damit wurden auch Versuche bezüglich der Darstellung "gemischter» Chinhydrone aus Homogentisinsäure + Benzochinon, sowie aus Benzohydrochinon + Benzochinonessigsäure angestellt.

1) Monatsh. f. Chemie, Bd. 28 (1907), S. 299. 


\section{Orientierende Versuche.}

Versuchsserie $A$. Die Versuche bezweckten in erster Linie, zu untersuchen, ob Chinhydronbildung in wässeriger Lösung der interessierenden Substanzen zustande kommen könne. Das Resultat war völlig negativ (keine Ausscheidung von Chinhydronkrystallen) in den sämtlichen 3 betreffenden Kombinationen. Dagegen wurde, abseits von der Hauptfrage, eine Beobachtung gemacht, die nicht ihres Interesses entbehrt. Bei der Arbeit mit äquimolekularen Mengen (bei Zimmerwärme) zeigte es sich nämlich, daß nach Verlauf von 24 Stunden die beiden Mischungen

1. Homogentisinsäure $(0,336 \mathrm{~g})+$ Benzochinon $(0,216 \mathrm{~g})$ $+30 \mathrm{ccm}$ Wasser,

2. Benzohydrochinon $(0,220 \mathrm{~g})+$ Benzochinonessigsäure $(0,332 \mathrm{~g})+30 \mathrm{ccm}$ Wasser

sich auf dieselbe. Weise verhalten. Aus beiden kann nun Benzohydrochinon in reichlicher Menge isoliert werden, während in der Mischung 1 weder Homogentisinsäure noch Benzochinon (außer einer unbedeutenden Spur) weiter nachgewiesen werden können. Die Art der Umsetzung in der Mischung 1 ist offenbar die, daß Benzochinon - während es selbst zu Benzohydrochinon reduziert wird - Homogentisinsäure zu Benzochinonessigsäure oxydiert. Mit anderen Worten, das System Benzohydrochinon-Benzochinonessigsäure ist in wässeriger Lösung stabiler als das System Homogentisinsäure-Benzochinon. Dies gilt, wie gesagt, für wässerige Lösung; in versuchten organischen Lösungsmitteln herrscht ein in gewissem Grade entgegengesetztes Verhältnis (siehe S. 323).

Versuchsserie $B$. In dieser, wie auch in der nächstfolgenden Serie wurde die Kombination Benzohydrochinon-Benzochinon, die notorische Chinhydronbildung herbeiführt, als Kontrollprobe verwendet. Die betreffenden Substanzen in annähernd äquimolekularen Mengen, wurden sorgfältig in Achatschale verrieben; die Farbe der Mischung wurde teils sofort (1), teils nach einem einzigen, leichten "Anhauchen» (2) notiert: 
1.

2.

a) Homogentisinsäure +Benzochinonessigsäure

blauviolett

kräftigere Färbung als in 1

b) Homogentisinsäure + Benzochinon

c) Benzohydrochinon

violettblau kräftigere Färbung als in 1 +Benzochinonessigsäure f Färbung

d) (Kontrolle) Benzohydrochinon + Benzochinon

violettblau

Für sämtliche Kombinationen gibt sich also wenigstens partielle Chinhydronbildung durch eintretende Färbung zu erkennen.

Versuchsserie $C$. In zwei Glasglocken wurde eine größere Portion von Chinonsubstanzen gestellt, in die eine (1) Benzochinon, in die andere (2) Benzochinonessigsäure. In jede der beiden Glasglocken wurden außerdem, in Uhrgläsern fein zerrieben, die beiden Hydrochinonsubstanzen eingestellt. Beobachtung nach 24 Stunden.

Glasglocke 1.

a) Homogentisinsäure, dunkel violettblau.

b) Benzohydrochinon, grauschwarz. ${ }^{1}$ )

Glasglocke 2.

a) Homogentisinsäure, keine Andeutung von Färbung (nicht einmal nach 5 Tagen).

b) Benzohydrochinon, dasselbe.

Die verschiedenen Resultate in den beiden Glasglocken stehen natürlich mit dem verschiedenen Verhalten der beiden Chinonsubstanzen bezüglich der Flüchtigkeit ${ }^{2}$ ) in Zusammenhang. Auch bei dieser Versuchsordnung gibt sich wenigstens partielle Chinhydronbildung für die Kombinationen HomogentisinsäureBenzochinon und Benzohydrochinon-Benzochinon zu erkennen.

1) Folgende Hydrochinonsubstanzen verhielten sich dagegen absolut negativ: Homogentisinsäurelacton, Hydrochinondimethyläther und Arbutin.

2) Die vorher, durch die Abwesenheit von Geruch und durch die Gewichtskonstanz während längere Zeit dauernder Aufbewahrung im $\mathrm{H}_{2} \mathrm{SO}_{4}$-Exsikkator, beobachtete Nichtflüchtigkeit der Benzochinonessigsäure erhält durch den eben beschriebenen Versuch eine weitere Bestätigung. 
Versuchsserie D. Bei Versetzen einer Ätherlösung der betreffenden Substanzen mit Ligroin oder einer Acetonlösung mit Benzol kam es, entweder direkt oder nach partieller Verdampfung im Wasserbade, bei allen drei Kombinationen:

Homogentisinsäure + Benzochinonessigsäure,

+ Benzochinon,

Benzohydrochinon + Benzochinonessigsäure,

zur Ausscheidung dunkler, nach Auspressen metallisch glänzender Krystallnadeln (= Chinhydronbildung).

2. Darstellung des «eigenen* Chinhydrons der Homogentisinsäure.

Versuch 1. 1,012 g Homogentisinsäure wurden gelöst in $125 \mathrm{ccm}$ Äther, $1,000 \mathrm{~g}$ Benzochinonessigsäure in $75 \mathrm{ccm}$ Äther, worauf gemischt und $200 \mathrm{ccm}$ Ligroin zugesetzt wurden. Binnen einigen Minuten begannen (bei Zimmerwärme) dunkle Krystallnadeln sich auszuscheiden und Boden und Wände des Gefäßes zu bekleiden. Die Mischung wurde bei glinder Wasserbadwärme auf ca. 1/4 Volumen konzentriert, die ausgeschiedene Substanz abfiltriert, ausgepreßt und dann erst offen an der Luft bei $0^{\circ}$, dann im $\mathrm{H}_{2} \mathrm{SO}_{4}$-Exsikkator bei $+30^{\circ}$ getrocknet. Ausbeute: $1,83 \mathrm{~g}$ (entsprechend $91 \%$ der theoretisch möglichen). Umkrystallisierung durch Auflösung in $450 \mathrm{ccm}$ warmem Äther, Zusatz von $100 \mathrm{ccm}$ Ligroin und Konzentrierung auf ca. $50 \mathrm{ccm}$ Volumen; die weitere Behandlung gleich der oben angegebenen. Ausbeute: $1,68 \mathrm{~g}=$ Präparat $\mathrm{I}$. Ein Teil davon wurde bei Zimmerwärme aus Aceton + Benzol umkrystallisiert = Präparat II.

Versuch 2. 0,506 g Homogentisinsäure wurden gelöst in $4 \mathrm{ccm}$ Aceton, $0,500 \mathrm{~g}$ Benzochinonessigsäure in $6 \mathrm{ccm}$ Aceton, nach Mischen wurden $75 \mathrm{ccm}$ Benzol hinzugesetzt, wobei, bei Zimmerwärme, fast sofort reichliche Ausscheidung von dunklen Mikrokrystallen eintrat. Diese wurden am folgenden Tage abfiltriert und wie oben behandelt. Ausbeute : 0,82 g (entsprechend $82 \%$ der theoretisch möglichen) = Präparat III.

Sämtliche Präparate - schön metallisch glänzend, nahezu schwarz mit einem Stich ins Olivbraune - bestanden aus mikroskopisch kleinen, breiten und dünnen Nadeln oder dünnen 
rhomboiden Tafeln; bei mikroskopischer Untersuchung erweist sich die Mehrzahl als undurchsichtig, nur die allerdünnsten lassen Licht durch, die Farbe dann rotbraun mit einem Stich ins Violette. Bei feinem Zerreiben erhält man ein dunkel blauviolettes Pulver. Schmelz-(Zersetzungs-)punkt für die drei Präparate: $+144,142$ bezw. $\left.142^{\circ} .^{1}\right)$

Qualitative Untersuchung.

Eine Portion wurde mit etwas Wasser geschüttelt, wobei bald eine rotgelbe Lösung erhalten wurde, die in 3 Portionen geteilt wurde.

Portion 1 wurde sofort mit neutraler Bleiacetatlösung versetzt - bald Krystalle von homogentisinsaurem Blei.

Portion 2 wurde sofort mit Chloroform ausgeschüttelt:

a) Das Chloroform gab bei Verdampfung bleijodidähnliche (nicht nach Chinon riechende) Krystalle von Benzochinonessigsäure;

b) die wässerige Lösung verhielt sich, bei Zusatz von neutraler Bleiacetatlösung, wie die Portion 1 oben.

Portion 3 wurde mit Wasser destilliert; das Destillat farb-, geruch- und geschmacklos (also kein Benzochinon enthaltend).

Elementaranalyse.

Präparat I. $0,1508 \mathrm{~g}$ gaben $0,3186 \mathrm{~g} \mathrm{CO}_{2}, 0,0588 \mathrm{~g} \mathrm{H}_{\mathbf{2}} \mathrm{O}$, waraus sich berechnen: 57,62\% $\mathrm{C}$ bezw. $4,37 \% \mathrm{H}$.

\begin{tabular}{ccc}
\multicolumn{2}{c}{ Gefunden: } & Berechnet für $\mathrm{C}_{16} \mathrm{H}_{14} \mathrm{O}_{8}$ : \\
C & $57,62 \%$ & $57,46 \%$ \\
$\mathrm{H}$ & $4,37 \%$ & $4,23 \%$ \\
$\mathrm{O}$ & $38,01 \%$ & $38,31 \%$
\end{tabular}

Durch Anwendung geeigneter organischer Lösungsmittel als Medien ist es also gelungen, der Berechnung gemäß und dazu mit guter Ausbeute, das «eigene» Ghinhydron der Homogentisinsäure :

$$
\left\{\begin{array}{l}
\mathrm{C}_{6} \mathrm{H}_{3}(\mathrm{OH})_{2} \cdot \mathrm{CH}_{2} \cdot \mathrm{COOH} \\
\mathrm{C}_{6} \mathrm{H}_{3} \mathrm{O}_{2} \cdot \mathrm{CH}_{2} \cdot \mathrm{COOH}
\end{array}\right.
$$

1) Für gewöhnliches Chinhydron wird $+171^{\circ} \mathrm{C}$. angegeben (Klinger und Standke, Ber. d. Deutsch. chem. Ges., Bd. 24 [1891], S. 1341). 
darzustellen. Es dürfte das zuerst dargestellte Chinhydron sein, in welchem die Hydrochinon- bezw. Chinonkonstituente carboxyl-substituiert sind.

3. Versuch, "gemischte» Chinhydrone von Homogentisinsäure usw. darzustellen.

Bei diesen Versuchen ist auf eine der bei der Darstellung des « eigenen • Chinhydrons der Homogentisinsäure angewandten analoge Weise mit den beiden Kombinationen:

A. Homogentisinsäure + Benzochinon (4 Präparate dargestellt),

B. Benzohydrochinon + Benzochinonessigsäure (4 Präparate dargestellt) (die betr. Substanzen in äquimolekularen Mengen verwendet) gearbeitet worden. Es sei gleich von vornherein bemerkt, daß die erhaltenen Reaktionsprodukte der Hauptsache nach nicht •gemischte Chinhydrone der Art waren, wie man es auf Grund der Art der angewandten Substanzen hätte erwarten können. Vielmehr hat sich bei qualitativer Prüfung und bei Elementaranalyse gezeigt, daß die erhaltenen Chinhydronprodukte der Hauptsache nach (wenn auch nicht in völlig reinem Zustande) dasselbe Chinhydron repräsentieren, das aus Homogentisinsäure + Benzochinonessigsäure erhalten worden war, d. h. das xeigene, Chinhydron der Homogentisinsäure!

Sowohl aus den A- als aus den B-Präparaten kann nämlich Homogentisinsäure in reichlicher Menge isoliert werden. Die erhaltenen Schmelzpunkte sind auch nicht in höherem Grade verschieden von den für das * eigene * Chinhydron der Homogentisinsäure gefundenen $\left(+142-144^{\circ}\right)$ :

für die A-Präparate: + 145, 143, 141 bezw. 1410,

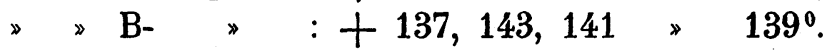

Von entscheidender Bedeutung für dịe Klarstellung der Frage ist in diesem Falle auch das Resultat der Elementaranalyse, nämlich hinsichtlich der C-Werte. 


\section{A-Präparate.}

Präparat II. $0,1634 \mathrm{~g}$ gaben $0,3475 \mathrm{~g} \mathrm{CO}_{2}, 0,0682 \mathrm{~g} \mathrm{H}_{2} \mathrm{O}$, woraus sich berechnen: 58,00\% C bezw. $4,67 \% \mathrm{H}$.

Präparat III. $0,1532 \mathrm{~g}$ gaben $0,3240 \mathrm{~g} \mathrm{CO}_{2}, 0,0596 \mathrm{~g} \mathrm{H}_{2} \mathrm{O}$, woraus sich berechnen: $57,68 \% \mathrm{C}$ bezw. $4,36 \% \mathrm{H}$.

\section{B-Präparate.}

Präparat II. $0,1468 \mathrm{~g}$ gaben $0,3118 \mathrm{~g} \mathrm{CO}_{2}, 0,0588 \mathrm{~g} \mathrm{H}_{2} \mathrm{O}$, woraus sich berechnen: 57,93\% C bezw. $4,49 \% \mathrm{H}$.

Präparat III. $0,1236 \mathrm{~g}$ gaben $0,2614 \mathrm{~g} \mathrm{CO}_{2}, 0,0491 \mathrm{~g} \mathrm{H}_{2} \mathrm{O}$, woraus sich berechnen: 57,68\% C bezw. $4,45 \% \mathrm{H}$.

Der in den Präparaten konstatierte C-Prozentgehalt beträgt demnach durchschnittlich:

während für das "eigene» Chinhydron der Homogentisinsäure sich berechnet:

und für «gemischtes» Chinhydron aus Homogentisinsäure + Benzochinon oder aus Benzohydrochinon + Benzochinonessigsäure :

57,46 ,

Offenbar hat in den betreffenden Reaktionsmischungen in wesentlicher, wenn auch nur partieller ${ }^{1}$ ) Ausdehnung, eine Umlagerung stattgefunden, so daß bei der A-Kombination Benzochinonessigsäure, bei der B-Kombination Homogentisinsäure neugebildet worden ist, wonach in beiden Fällen Chinhydronbildung zwischen in der Mischung disponibler Homogentisinsäure und Benzochinonessigsäure stattgefunden hat.

\section{Verhalten der Homogentisinsäure gegen Ferrichlorid (bei gew. Temperatur).}

Wie allgemein bekannt, ruft Zusatz von Ferrichloridlösung zu einer wässerigen Lösung von Homogentisinsäure eine vorübergehende Blaufärbung hervor. Beim Arbeiten mit einer nicht allzu schwachen Homogentisinsäure- bezw. nicht allzu kon-

1) In Übereinstimmung hiermit hat die Chinhydronausbeute bezüglich des Gewichts bei diesen Versuchen sich weit weniger günstig gestellt als bei der absichtlichen Darstellung des e eigenen, Chinhydrons der Homogentisinsäure. 
zentrierten Ferrichloridlösung, unter allmählich geschehendem Zusatz der letzteren, beobachtet man, daß nach dem Verschwinden der zuerst entstandenen Blaufärbung eine neue solche bei weiterem Ferrichloridzusatz auftritt. Dies jedoch nur bis $\mathrm{zu}$ einer gewissen Grenze, wonach erneuter Ferrichloridzusatz. ohne sichtbare Wirkung in der genannten Hinsicht ist. Natürlich beruht dieses Verhalten teils darauf, daß das Ferrisalz zu Ferrosalz (das nicht mit Homogentisinsäure reagiert) reduziert wird, während Homogentisinsäure in entsprechendem Maße oxydiert wird, teils darauf, daß schließlich keine unveränderte Homogentisinsäure mehr übrig ist. Meine Absicht war nun, festzustellen, ob auch das bei dieser Reaktion entstehende 0xydationsprodukt der Homogentisinsäure mit der auf anderem Wege gewonnenen Benzochinonessigsäure identisch sei. Daß . dies der Fall ist, läßt sich ohne Schwierigkeit konstatieren. $1,0 \mathrm{~g}$ Homogentisinsäure $+20 \mathrm{ccm}$ Wasser wurden bei Zimmerwärme mit $40 \mathrm{ccm}$ Ferrichloridlösung (25\% kryst. Salz enthaltend) versetzt, wobei die Mischung zuerst blauschwarze, dann dunkel braunrote, schließlich nur braungelbe Farbe zeigte. Die Mischung wurde nach 1/4 Stunde mit Äther ausgeschüttelt. Der ausgewässerte, goldgelbe Äther wurde auf einige wenige Kubikzentimeter Volumen eingedampft, wobei schön glänzende, gelbe Krystalltafeln sich ausschieden, die, nach Isolierung, leicht als Benzochinonessigsäure (u. a. Schmelzpunkt: $+131^{\circ}$ ) identifiziert wurden. ${ }^{1}$ )

In einem anderen Versuch, der in allen Hinsichten gleich dem vorhergehenden angeordnet wurde, jedoch mit dem Unterschiede, daß die Ferrichlorideinwirkung weit schwächer wurde (Verwendung von 5\% iger Ferrichloridlösung, Ausschütteln mit Äther unmittelbar nach dem Hinzusetzen), gestaltete sich das

1) Gegenwart von Benzochinon oder einer anderen, *Chinongeruch von sich gebenden Substanz wurde dagegen nicht wahrgenommen. Über eine im Gange befindliche Nachprüfung der Angabe Wolkow und Baumanns, daß beim Kochen von Homogentisinsäure mit konzentrierter Ferrichloridlösung Chinongeruch auftrete (welche Prüfung noch nicht zum Abschluß gekommen ist, da der verfügbare Vorrat von Homogentisinsäure ein Ende genommen hat), wird später berichtet werden. 
Resultat dem Anschein nach verschieden - wenn auch in Prinzip gleichartig. Der Äther gab nämlich beim Verdampfn einen braunvioletten, metallisch schimmernden Rückstard (= Gegenwart des * eigenen» Chinhydrons der Homogentisilsäure), was darauf beruhte, daß der Äther aus der nicht volständig oxydierten Reaktionsmischung ${ }^{1}$ ) außer Benzochino1essigsäure auch unveränderte Homogentisinsäure aufgenommm hatte.

1) Diese war in diesem Versuch noch von dunkel braunroter Farje (vgl. oben!). 